\title{
The Pulfrich phenomenon and its alleviation with a neutral density filter
}

\author{
GORDON HERON' AND GORDON N DUTTON² \\ From the 'Department of Ophthalmic Optics, Glasgow College of Technology, Cowcaddens Road, Glasgow \\ G4 OBA, and the 'Tennent Institute of Ophthalmology, Western Infirmary, Glasgow G11 6NT
}

SUMMARY A case is described in which a presumed vascular accident resulted in long-standing visual difficulties in both reading and the analysis of vectors of moving objects. Clinical examination revealed minimal right optic atrophy with a relative superior altitudinal visual field defect associated with a positive Pulfrich effect. A partial head turn to the right in association with paresis of saccades and pursuit eye movements to the right was also evident. Spectacles for distance which incorporated a neutral density filter before the left eye were prescribed. These practically eliminated the Pulfrich effect and alleviated the problems of vector analysis. A near correction was provided which incorporated prisms with bases to the right. This eliminated the reading difficulties. The visual problems experienced by patients suffering from a positive Pulfrich effect are described and discussed.

The Pulfrich effect or stereophenomenon' is a disturbance in the perception of a moving object brought about by a difference in the time taken for the image data from each eye to be registered in the occipital cortex. When one or the other eye is covered by a neutral density filter, an object typically a pendulum bob, moving at right angles to the line of sight - that is, in the fronto-parallel plane (FPP) - appears to move in an elliptical path. The relative delay in perception for one eye creates slightly different apparent locations in space for the images from each eye. This difference is registered cortically as a geometric disparity which results in an incorrect analysis of the vector, giving the illusion the striking characteristic of apparent depth.

The phenomenon can either be elicited in normal observers by placing a neutral density filter in front of one eye (provoked Pulfrich effect), or occur as a result of pathological disturbance in optic nerve function (positive or spontaneous Pulfrich effect). The elliptical path of the pendulum appears clockwise when the filter is placed before the left eye and anticlockwise for the right eye (Fig. 1). The magnitude of the effect (that is the apparent size of the minor axis of the ellipse) is proportional to the density of filter used. The effect occurs independently

Correspondence to Mr Gordon Heron. of eye movements, and for the provoked effect the eyes track the actual rather than the apparent path of the pendulum. ${ }^{2}$

Emphasis has been given to the diagnostic value of the test, which investigates the effect in patients with optic nerve dysfunction, ${ }^{3-4}$ but little attention has been directed to the problems experienced by patients and whether or not these can be alleviated. We describe a patient whose symptoms of visual disturbance due to the Pulfrich effect were ameliorated by using a suitable filter before the unaffected eye, and for whom the prescription of appropriate prisms overcame reading difficulties due to paresis of saccadic and pursuit eye movements to the right.

\section{Case report}

The patient, born on 6 December 1937, was a housewife who was first seen by one of us (GD) in December 1984. Three years earlier she had undergone surgery to the temporomandibular joint under general anaesthesia. She had woken from surgery with weakness of the right arm and leg, right upper motor neurone facial palsy, difficulty in looking to the right, right upper quadrantic homonymous visual field loss, and impairment in vision of the right eye. However, there was no gaze evoked nystagmus, no lateropulsion could be elicited, and 'Doll's head' eye 


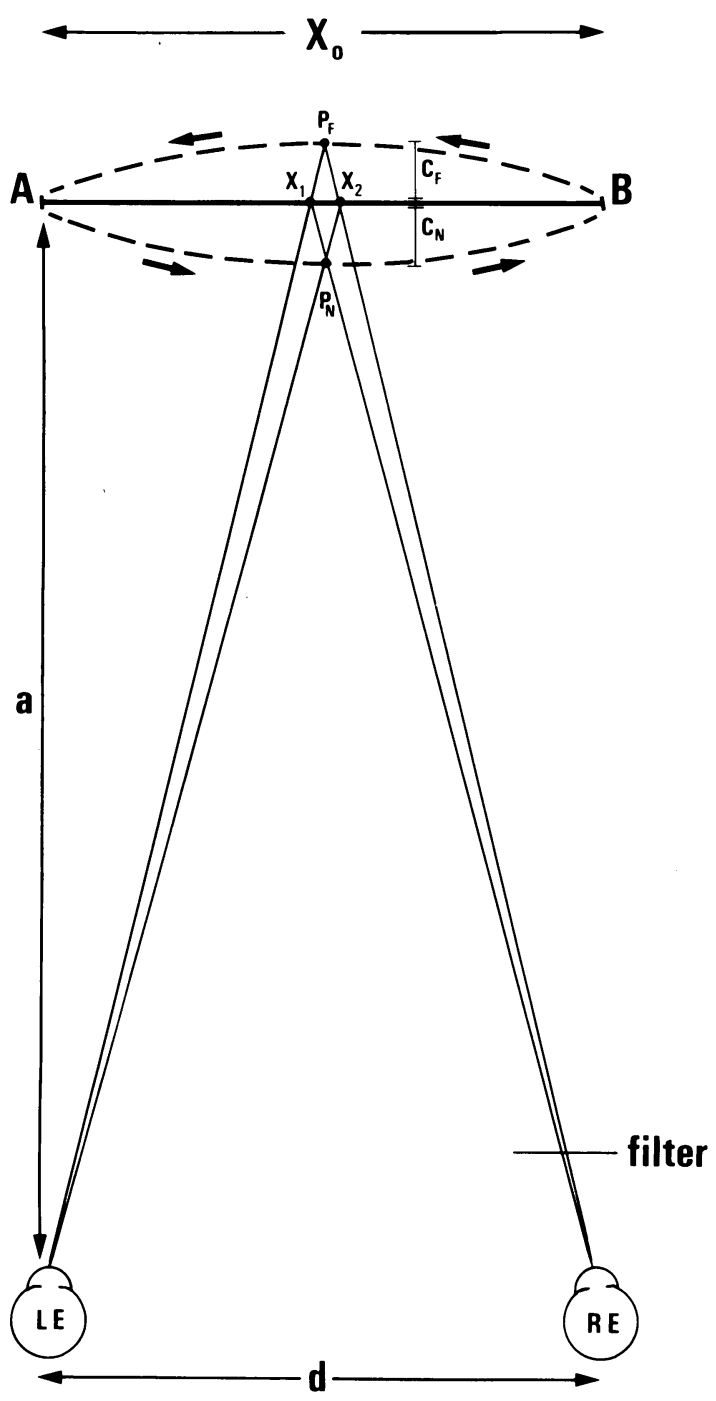

\section{- - - apparent path of target actual path of target}

Fig. 1. The provoked Pulfrich effect: diagram of the apparent path of target moving with a simple harmonic motion along the plane $A B$, when a filter is placed before the right eye.

movements were normal. These symptoms and signs were attributed at the time to multifocal vascular accidents affecting the left cerebral hemisphere and the right optic nerve, which had followed a hypotensive episode during surgery. During the ensuing six months her limb and facial weakness and her homonymous field defect resolved spontaneously, but a subtle relative superior altitundinal visual field defect was still detectable for the right eye by Tübinger perimetry $(1.0 \mathrm{~W}, 100 \mathrm{Abs})$.

On presentation in 1984 the patient complained of two anomalies of her visuospatial environment, from which she had suffered from the time of her operation. Objects moving towards her from the right side appeared to swerve to approach her directly; she also experienced great difficulty in placing objects centrally, always placing them to her left.

When she was a passenger in a car on the motorway, cars on the opposite carriageway appeared to jump the crash barrier and approach her. Her most dramatic observation concerned her perception of a mechanical digger, which was moving in a field on her right while she was travelling in a car. As the car drove past, the digger appeared about to crash into the car and she took evasive action, pushing the driver to one side. The apparent height of doorways was also underestimated, and she felt that she had to duck her head when moving between rooms at home. Activities requiring near central vision were particularly difficult. Reading material was placed to the left for greatest clarity and she had abandoned embroidery, as she was unable to keep her stitches in line.

On examination she preferred to adopt a head turn to the right. Her corrected visual acuities were $6 / 9$ right $(+0 \cdot 50 /+0 \cdot 25$ at 170$)$ and $6 / 6$ left $(+0 \cdot 25 /+0 \cdot 25$ at 170). Examination of her eye movements revealed an impairment of both saccades and pursuits to the right; eye movements to the left were full and normal.

There was pallor of her right optic nerve head in association with the persistent right relative superior altitudinal visual field defect. Despite careful testing there was no clinically detectable afferent pupil deficit, and the pupil cycle time ${ }^{11}$ for three separate recordings for each eye was $0.72,0.70$, and $0.72 \mathrm{~s}$ right and $0.72,0.74$, and $0.72 \mathrm{~s} \mathrm{left}$. A pendulum was employed to elicit a Pulfrich phenomenon, and this was positive, the pendulum bob appearing to rotate in an anticlockwise fashion. Third generation computerised tomography did not demonstrate any intracerebral or optic nerve pathology. Pattern reversal visual evoked responses with $1.8^{\circ}$ check size gave latencies of $95 \pm 5 \mathrm{~ms}$ for each eye, with equal amplitudes of approximately $75 \%$ of the normal for our laboratory.

CLINICAL.METHODS*

$\mathrm{A}+2.50 \mathrm{DS}$ addition to each eye gave a combined near visual acuity of N12. Additional 6-dioptre

*Informed consent was gained from the patient for all the procedures described. 
A

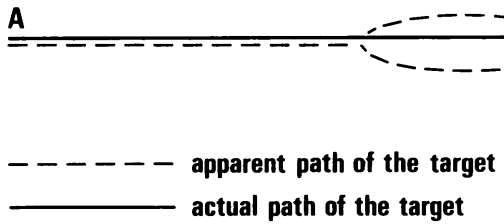

Fig. 2. The appearance of the target with a 0.2 ND filter placed before the left eye. The elliptical pattern of the spontaneous Pulfrich effect remained as a very shallow ellipse as the target moved into the right field and a straight line as the target moved out to the left field.

prisms with bases to her right improved her near acuity to N6, and also enabled the patient to hold reading material in the central position. Spectacles with this prescription were dispensed; these allowed her to read with ease and to perform embroidery accurately.

The Pulfrich phenomenon was initially investigated by a white target mounted on a metronome. The target appeared to move in an asymmetrical anticlockwise ellipse which was shallower when the target moved from right to left and was seen to be higher on the right than the left. The apparent FPP traversed by the target also appeared to tilt, being nearer on the right limit of excursion and further away on the left. A $0 \cdot 3$ neutral density (ND) filter placed before the left eye reversed the effect, and a $0 \cdot 2$ ND filter partially eliminated it. With the latter filter no ellipse was seen for target positions to her left, but a shallow ellipse was still present, but not tilted, for target positions to her right (Fig. 2). The $0 \cdot 2$ ND filter was initially taped to the left lens of the distance spectacles, and subsequently replaced by an ophthalmic tint (Norvac G33, Norville Optical) which was empirically selected on the basis of elimination of the Pulfrich effect. The luminous transmission factor of this ophthalmic tint was found to be slightly greater than a $0 \cdot 2$ ND filter.

When reviewed three months later the patient had found that the distance glasses had reduced the strange patterns of perception for both moving and for stationary objects. The filter was not required for near, since the illusion was experienced for distance targets only. This effect has been sustained for the ensuing three years. The patient has not noted the minor shifts in colour vision reported with prolonged use of a neutral filter ${ }^{11}$ and neither has there been any evidence of visual fatigue. ${ }^{12}$

\section{Discussion}

Although the visual evoked response (VER) had shown no difference in latency between the two eyes, the presence of the positive Pulfrich effect, which was reproducible, suggested that a small latency difference beyond the limits of detection by the VER was present. The magnitude of the Pulfrich effect was determined using an X-Y plotter modified so that its pen carriage would transcribe an ellipse as it traversed the $\mathrm{X}$ axis. The size of the ellipse (minor axis) could be varied, and a $12 \mathrm{~mm}$ white disc target was used, supported $20 \mathrm{~cm}$ above the pen carriage. The patient was asked to observe the target without spectacles, and the apparatus was adjusted until the target transcribed an ellipse which was not observed by the patient. $\mathrm{Lit}^{13}$ has given the equation which describes the time difference $(\Delta t)$ of a target to travel from $X_{1}$ to $X_{2}$ along the FPP. This is equal to the difference in the latency periods between the two eyes (Fig. 1).

$$
\begin{aligned}
& \Delta t=\frac{T\left(X_{1}-X_{2}\right) \times 10(0)}{2 \pi X_{0}} \\
& \text { where }\left(X_{1}-X_{2}\right)=\frac{d C_{n}}{a-C_{n}} \text { or } \frac{d C_{1}}{a+C_{1}}
\end{aligned}
$$

$T$ is the period and $X_{0}$ is the amplitude of the pendulum. For the arrangement described above, the ellipse which climinated the Pulfrich effect had an amplitude of $16.5 \mathrm{~cm}$, a period of 3 seconds, and a

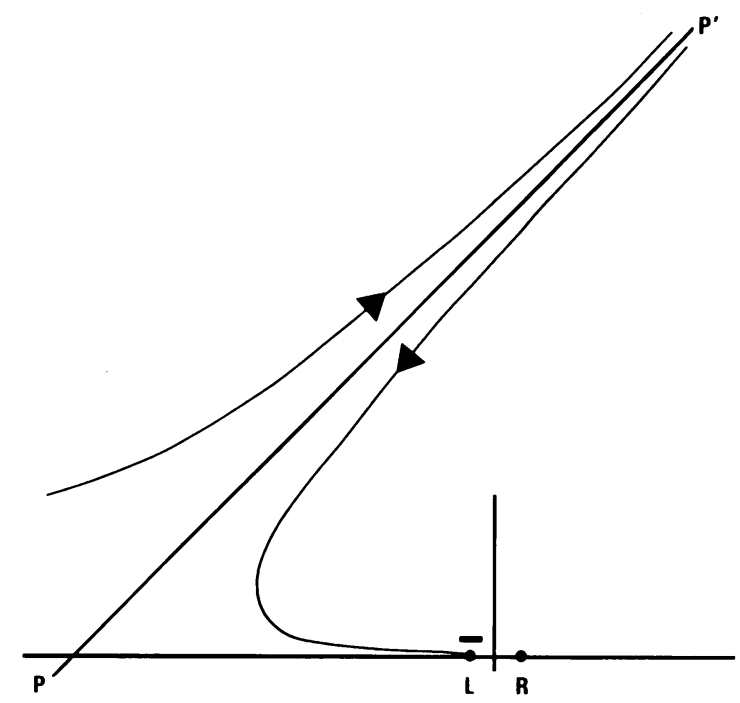

Fig. 3. Theoretical hyperbolas which describe the apparent path of a moving object along plane $P P^{\prime}$, for the Pulfrich effect provoked by a filter before the left eye. For object moving from left to right the path is described by the hyperbola above the plane (rightwards pointing arrow) and for objects moving from the right to left along $P P^{\prime}$ the apparent path is along the hyperbola below the plane (leftwards pointing arrow). Redrawn from Spiegler ${ }^{16}$ and reproduced with permission. 
minor axis of $2.38 \mathrm{~cm}$ when viewed from 1 metre. These figures result in a latency difference $(\Delta t)$ of 2.25 milliseconds. This value is outside the range of detectable latency difference for the VER.

Although it has been suggested previously that a neutral density filter would abolish the symptoms engendered by the Pulfrich effect, we are aware of only a single case in which this has been attempted. This was the first recorded case, but no follow-up was reported. ${ }^{14}$ Many of the bizarre symptoms that our patient reported have been recorded in previous studies on patients with demyelinating disease $e^{3 t-x}$ and have been replicated by experimental studies of the provoked Pulfrich effect in normal individuals. ${ }^{\circ} 15$ However, for an object moving in a straight path which obliquely intersects the interpupillary plane the apparent path has been shown theoretically to be hyperbolic (Fig. 3). Various hyperbolas are observed depending on the angle of intersection and whether the target approaches or recedes from the observer. ${ }^{16}$ This may explain our patient's localisation of moving objects (such as cars), and might also relate directly to her perception of doorways, in which the height was underestimated. In this case the object is stationary, and it is the observer who is moving. In addition the phenomenon of irradiation stereoscopy ${ }^{17}$ may be the reason that patients with a spontaneous Pulfrich effect notice that static objects appear tilted. However, brightly lit high reflectance objects are needed to produce the effect in the laboratory; hence the influence of irradiation stereoscopy in this case is uncertain.

Increased latency of the pattern reversal VER occurs in cases of optic neuritis. ${ }^{18}$ In a detailed study Rushton ${ }^{19}$ has compared this with the delays determined from the associated Pulfrich effect. Although the presence of a Pulfrich effect correlated well with the detection of increased latency, there was no correlation between the latency values determined by the two methods. Rushton suggests that this may have been due to small tracking variations as patients followed the moving target, since foveal and nonfoveal locations have differing latencies. This may have occurred with our patient, since the residual ellipse observed by her when the neutral density filter was in place was seen on right gaze, the direction of the gaze paresis.

We presume that a multifocal ischaemic episode caused the symptoms and signs. The relative hemiparesis and facial palsy could be accounted for on the basis of a vascular lesion in the contralateral basal pontine region. This could also have affected the pontine paramedian reticular formation and thus given rise to the permanent dysfunction in horizontal gaze. In addition, impaired vision of the right eye implicates right optic nerve dysfunction, thus implicating the internal carotid artery system. However, lesions in the medulla oblongata can be discounted, since there are no symptoms consistent with those typically encountered in Wallenberg's Syndrome, ${ }^{21}$ namely lateropulsion, imbalance, and tilting of the visual world.

The symptoms of anomalous perception described by our patient can easily be ignored or dismissed if the clinician is unaware of the Pulfrich phenomenon. The condition can be both disturbing and disabling. This case highlights the problems which such patients experience and demonstrates that an appropriate neutral density filter before the least affected eye can bring considerable long-term symptomatic relief.

We gratefully acknowledge the assistance given by colleagues in these investigations. R G Ackerley, J R Pugh, and A S Eadie assisted with the generation of ellipses on the XY plotter and with the recording of eye movements. D Allan recorded the VER and eye movements at the Western Infirmary, Glasgow.

\section{References}

1 Pulfrich C. Die Stereoskopie im Dienste det Isochromen und Heterochromen Photometrie. Naturwissenschaften 1922; 10: 553-64, 569-601, 714-22, 735-43, 751-61.

2 Ono H, Steinbach MJ. The Pulfrich phenomenon with eye movement. Vision Res 1983; 23: 1735-7.

3 Sach E. Abnormal delay of visual perception. Arch Neurol Psychiatry 1946; 56: 198-206.

4 Frisen L, Hoyt WF, Bird AC, Weale RA. Diagnostic uses of the Pulfrich phenomenon. Lancet 1973; ii: 385-6.

5 Sokol S. The Pulfrich stereo-illusion as an index of optic nerve dysfunction. Surv Ophthalmol 1976; 20: 432-4.

6 Salgsvold JE. Pulfrich pendulum phenomenon in patients with a history of acute optic neuritis. Acta Ophthalmol (Kbh) 1978; 56: $817-26$.

7 Lanthony P. Le Phénomène de Pulfrich.J Fr Ophtalmol 1984; 7 : 575-87.

8 Wertenbaker C, Gutman I. Unusual visual symptoms. Surv Ophthalmol 1985; 29: 297-9.

9 Bird AC, Leaver PK, Gould E, McDonald I. Optic neuritis: its natural course and evaluation of therapy. Presented to the Symposium of Neuro-Ophthalmology, Bascom, Palmer Eye Institute, Univ. Miami, Florida, 1974.

10 Miller SD, Thompson HS. Edge-light pupil cycle time. $\mathrm{Br} \mathrm{J}$ Ophthalmol 1978; 62: 495-500.

11 Hill AR, Stevenson RWW. Long-term adaptation to ophthalmic tinted lenses. Col. vis. def. III. Mod Probl Ophthalmol 1976; 17: $264-72$.

12 Tagami Y, Ohnuma T, Isayama Y. Visual fatigue phenomenon and prescribing tinted lenses in patients with optic neuritis. Br J Ophthalmol 1984; 68: 208-11.

13 Lit A. The magnitude of the Pulfrich stereophenomenon as a function of binocular differences of intensity at various levels of illumination. Am J Psychol 1949; 62: 159-81.

14 Grimsdale H. A note on Pulfrich's phenomenon with a suggestion on the possible clinical importance. Br J Ophthalmol 1925:9: 63-5.

15 Wilson GS. An investigation of the Pulfrich effect. Br J Physiol Opt 1965; 22: 208-37.

16 Spiegler JB. Apparant path of a Pulfrich target as a function of the slope of its plane of motion: a theoretical note. Am J Optom Physiol Opt 1986; 63: 209-16.

17 Ogle KN. The optical space sense. In: Davson H, ed. The eye London: Academic Press, 1962: 4: 302-3. 
18 Halliday AM. McDonald WI, Mushin J. Delayed visual evoked response in optic neuritis. Lancet 1972; i: 982-5.

19 Rushton D. Use of the Pulfrich pendulum for detecting abnormal delay in the visual pathway in multiple sclerosis. Brain 1975: 98: 283-96.
20 Hornsten G. Wallenberg's syndrome. Acta Neurol Scand 1974: $50434-46$.

Accepted for publication 30 June 1989 